\title{
Local Integral Estimates for Quasilinear Equations with Measure Data
}

\author{
Qiaoyu Tian, ${ }^{1}$ Shengzhi Zhang, ${ }^{2}$ Yonglin $\mathrm{Xu}^{1}{ }^{1}$ and Jia $\mathrm{Mu}^{1}$ \\ ${ }^{1}$ School of Mathematics and Computer, Northwest University for Nationalities, Lanzhou, Gansu 730030, China \\ ${ }^{2}$ Department of Mathematics, Gansu Normal University for Nationalities, Hezuo, Gansu 747000, China
}

Correspondence should be addressed to Qiaoyu Tian; tianqiaoyu2004@163.com

Received 16 February 2016; Accepted 24 April 2016

Academic Editor: Alberto Fiorenza

Copyright (C) 2016 Qiaoyu Tian et al. This is an open access article distributed under the Creative Commons Attribution License, which permits unrestricted use, distribution, and reproduction in any medium, provided the original work is properly cited.

Local integral estimates as well as local nonexistence results for a class of quasilinear equations $-\Delta_{p} u=\sigma P(u)+\omega$ for $p>1$ and Hessian equations $F_{k}[-u]=\sigma P(u)+\omega$ were established, where $\sigma$ is a nonnegative locally integrable function or, more generally, a locally finite measure, $\omega$ is a positive Radon measure, and $P(u) \sim \exp \alpha u^{\beta}$ with $\alpha>0$ and $\beta \geq 1$ or $P(u)=u^{p-1}$.

\section{Introduction and Main Results}

Let $\Omega \subset \mathbb{R}^{N}$ be a bounded domain, let $\sigma$ be a nonnegative locally integrable function or, more generally, a locally finite measure on $\Omega$, and let $\omega$ be a nonnegative Borel measure. In this paper, we consider the following nonlinear partial differential equations with measure data:

$$
-\Delta_{p} u=\sigma P(u)+\omega
$$

$$
u \geq 0, u \text { is } p \text {-superharmonic in } \Omega \text {, }
$$

where $P(u)=P_{\ell, \alpha, \beta}(u) \in L_{\sigma, \text { loc }}^{1}(\Omega)$ is defined, following $[1,2]$, as

$$
P_{\ell, \alpha, \beta}(u)=H_{\ell}\left(\alpha u^{\beta}\right)
$$

and $\ell$-truncated exponential function $H_{\ell}(r)$ is given by

$$
H_{\ell}(r)=\sum_{j=\ell}^{\infty} \frac{r^{j}}{j !} .
$$

Here, $\Delta_{p} u$ is the $p$-Laplacian of $u$ defined by $\Delta_{p} u$ := $\operatorname{div}\left(|D u|^{p-2} D u\right)(p>1)$. For convenience, here and elsewhere in the paper, we assume that $\ell \beta>p-1$. We will understand (1) in the following potential-theoretic sense using $p$-superharmonic functions (see Section 2).
Quasilinear equations (1) where $\sigma P(u)$ term is replaced by $\sigma u^{q}$ for superlinear case $q>p-1$ are well studied (see [3$5]$ and references therein, see [6,7] for natural growth terms $q=p-1$, and see $[8,9]$ for sublinear problems $q<p-1)$. In particular, it was shown in [3] that if $u \in L_{\sigma, \text { loc }}^{q}(\Omega)$ is a solution of (1) with nonlinear source terms $\sigma u^{q}, q>p-1$ in $\Omega$ and $B_{4 R} \subset \Omega$, then there is a constant $M=M(N, p, q)$ such that

$$
\begin{aligned}
& \left\{\int_{0}^{r}\left[\frac{\sigma\left(B_{t}\right)}{t^{N-p}}\right]^{1 /(p-1)} \frac{d t}{t}\right\}^{(p-1) /(q-p+1)} \\
& \cdot \int_{r}^{R / 2}\left[\frac{\int_{B_{t}} u^{q} d \sigma}{t^{N-p}}\right]^{1 /(p-1)} \frac{d t}{t} \leq M,
\end{aligned}
$$

for all $0<r \leq R / 4 ;(4)$ improves the related results of $[10,11]$.

Recently, Quoc-Hung and Véron [2] obtained twosided estimates on the solutions in terms of $T$-truncated $\eta$-fractional maximal potential of $\omega$, which is suitable for dealing with exponential nonlinearities:

$$
M_{\alpha, T}^{\eta}=\sup \left\{\frac{\omega\left(B_{t}\right)}{t^{N-\alpha} h_{\eta}(t)}: 0<t \leq T\right\},
$$




$$
\begin{aligned}
& \text { where } \\
& h_{\eta}(t) \\
& = \begin{cases}(-\ln t)^{-\eta} \chi_{(0,1 / 2]}(t)+(\ln 2)^{-\eta} \chi_{[1 / 2,+\infty)}(t), & \eta>0, \\
1, & \eta=0 .\end{cases}
\end{aligned}
$$

Some analogous estimates for Hessian equations also are given in this paper.

In this paper, firstly, we will establish a priori estimates of (1) with exponential reaction $P_{\ell, \alpha, \beta}(u)$, defined by (2) and (3). One of our main results are the following theorems.

Theorem 1. Let $u(x)$ be a solution of (1) in $\Omega$ with $p>1$ and $\ell \beta>p-1$. Suppose that $B_{4 R}\left(x_{0}\right) \subset \Omega$. Then, there exists a constant $M=M(N, p, \alpha, \beta, \ell)$ such that

$$
\begin{gathered}
\left\{\int_{0}^{r}\left[\frac{\sigma\left(B_{t}\right)}{t^{N-p}}\right]^{1 /(p-1)} \frac{d t}{t}\right\}^{(p-1) /(\ell \beta-p+1)} \\
\cdot \int_{r}^{R / 2}\left[\frac{\omega\left(B_{t}\left(x_{0}\right)\right)}{t^{N-p}}\right]^{1 /(p-1)} \frac{d t}{t} \leq M, \\
\left\{\int_{0}^{r}\left[\frac{\sigma\left(B_{t}\right)}{t^{N-p}}\right]^{1 /(p-1)} \frac{d t}{t}\right\}^{(p-1) /(\ell \beta-p+1)} \\
\cdot \int_{r}^{R / 2}\left[\frac{\int_{B_{t}} P_{\ell, \alpha, \beta}(u) d \sigma}{t^{N-p}}\right]^{1 /(p-1)} \frac{d t}{t} \leq M,
\end{gathered}
$$

for all $0<r \leq R / 4$.

As a consequence of Theorem 1, we have the following nonexistence results of local solutions to quasilinear equations.

Theorem 2. Let $u$ be a solution of (1) in an open connected set $\Omega \subset \mathbb{R}^{N}$. Suppose that $1<p<n, d \sigma=|x|^{-r} d x$ with $r>p$, and $0 \in \Omega$. Then, $u \equiv 0$.

Now, we consider (1) with natural growth terms; that is, the $\sigma P(u)$ term in $(1)$ is replaced by $\sigma u^{p-1}$. It is worthwhile to point out that this problem turns out to be more complex than the supercritical case. The interaction between the differential operator $-\Delta_{p} u$ and the lower order term $\sigma u^{p-1}$ was investigated by Jaye and Verbitsky $[6,7]$.

Theorem 3. Let $u(x)$ be a solution of (1) in $\Omega$ with $p>1$ and $P(u)=\sigma u^{p-1}$. Suppose that $B_{4 R}\left(x_{0}\right) \subset \Omega$. Then, there exists a constant $M=M(N, p, \alpha, \beta, \ell)$ such that

$$
\begin{aligned}
& \int_{0}^{r}\left[\frac{\sigma\left(B_{t}\right)}{t^{N-p}}\right]^{1 /(p-1)} \frac{d t}{t} \int_{r}^{R / 2}\left[\frac{\omega\left(B_{t}\left(x_{0}\right)\right)}{t^{N-p}}\right]^{1 /(p-1)} \frac{d t}{t} \\
& \quad \leq M, \\
& \int_{0}^{r}\left[\frac{\sigma\left(B_{t}\right)}{t^{N-p}}\right]^{1 /(p-1)} \frac{d t}{t} \int_{r}^{R / 2}\left[\frac{\int_{B_{t}} u^{p-1} d \sigma}{t^{N-p}}\right]^{1 /(p-1)} \frac{d t}{t} \\
& \quad \leq M,
\end{aligned}
$$

for all $0<r \leq R / 4$.
Similarly, we have the following.

Theorem 4. Let $u \in L_{l o c}^{p-1}(d \sigma)$ be a solution of $(1)$ with $P(u)=$ $\sigma u^{p-1}$ in an open connected set $\Omega \subset \mathbb{R}^{N}$. Suppose that $1<p<$ $n, d \sigma=|x|^{-p} d x$, and $0 \in \Omega$. Then, $u \equiv 0$.

Remark 5. The four previous theorems are particular case of the more general class of nonlinear Wolff integral equations:

$$
u(x)=\mathbf{W}_{\alpha, p}^{R}[P(u) d \sigma](x)+f(x),
$$

where $f(x)=\mathbf{W}_{\alpha, p}^{R}[\omega](x)$, which includes fractional Laplacian $(-\Delta)^{\gamma}$. Therefore, we also can obtain similar results of these integral equations.

The plan of the paper is as follows. In Section 2, we collect some elements notions and potential estimates for $p$ superharmonic. Theorems 1 and 2 will be proved in Section 3 . In this section, we also discuss the relations of $\sigma$ and $\omega$ provided that there exist solutions of (1). After this, Section 4 presents the proof of Theorems 3 and 4 by a new iteration scheme. Section 5 is devoted to considering fully nonlinear analogues of the Dirichlet problem (1) for Hessian equations without proof.

\section{Preliminaries}

In this section, we first recall some notations and definitions. In the following, we denote by $C$ a general constant, possibly varying from line to line, to indicate a dependence of $C$ on the real parameters $N, p, \alpha, \beta, \ell$; we will write $C=$ $C(N, p, \alpha, \beta, \ell)$. We also denote by $B\left(x_{0}, r\right)=\left\{x \in \mathbb{R}^{n}\right.$ : $\left.\left|x-x_{0}\right|<r\right\}$ the open ball with center $x_{0}$ and radius $r>0$; when it is not important or clear from the context, we shall omit denoting the center as $B_{r}=B\left(x_{0}, r\right)$.

Let $p>0$ and $\sigma$ be a nonnegative Borel measures in $\Omega$ which are finite on compact subsets of $\Omega$. The $\sigma$-measure of a measurable set $E \subset \Omega$ is denoted by $\sigma(E):=\int_{E} d \sigma$. We denote by $L^{p}(\Omega, d \sigma)\left(L_{\text {loc }}^{p}(\Omega, d \sigma)\right.$, resp.) the space of measurable functions $f$ such that $|f|^{p}$ is integrable (locally integrable) with respect to $\sigma$. When $d \sigma=d x$, we write $L^{p}(\Omega)\left(L_{\text {loc }}^{p}(\Omega)\right.$, resp.).

For $\alpha>0, p>1$, such that $\alpha p<n$, the $R$-truncated Wolff's potential $\mathbf{W}_{1, p}^{R} \mu(x)$ of a nonnegative Borel measure $\mu$ on $\mathbb{R}^{N}$ is defined by

$$
\mathbf{W}_{1, p}^{R}[\mu](x)=\int_{0}^{R}\left(\frac{\mu\left(B_{t}\right)}{t^{N-p}}\right)^{1 /(p-1)} \frac{d t}{t} .
$$

We also denote by $\mathbf{W}_{1, p}[\mu](x)$ the $\infty$-truncated Wolff's potential.

In this paper, all solutions are understood in the potentialtheoretic sense. A lower semicontinuous function $u: \Omega \rightarrow$ $(-\infty,+\infty]$ is called $p$-superharmonic if $u$ is not identically infinite in each component of $\Omega$, and if for all open sets $D$ such that $\bar{D} \subset \Omega$, and all functions $h \in C(D)$, $p$-harmonic in $D$, the implication holds: $h \leq u$ on $\partial D$ implies $h \leq u$ in $D$. Note that $p$-superharmonic function $u$ does not necessarily 
belong to $W_{\text {loc }}^{1, p}(\Omega)$, but its truncation $T_{k}(u)=\min \{u, k\}$ does for every integer $k$; therefore, we will need the generalized gradient of a $p$-superharmonic function $u$ defined by $D u=$ $\lim _{k \rightarrow \infty} \nabla\left(T_{k}(u)\right)$. For more properties of $p$-superharmonic, see [12].

The following lower pointwise estimates for $p$ superharmonic functions play an important role in our estimate.

Proposition 6 (see [13]). There exists a positive constant $C=$ $C(N, p)$ such that if $u \geq 0$ is $p$-superharmonic on $\Omega$ and $\mu=$ $-\Delta_{p} u$, then

$$
\left[\frac{\mu\left(B_{R}\right)}{R^{N-p}}\right]^{1 /(p-1)} \leq C \inf _{B_{R}} u
$$

for all balls $B_{R}$ such that $B_{2 R} \subset \Omega$.

The following lemma was also proved in [13].

Proposition 7. Let $B_{R}(x)$ be a ball such that $B_{2 R}(x) \subset \Omega$. Then, there exists a positive constant $C=C(N, p)$ such that if $u \geq 0$ is $p$-superharmonic on $\Omega$ and $\mu=-\Delta_{p} u$, then

$$
C \mathbf{W}_{1, p}^{R}[\mu](x) \leq u(x),
$$

where $\mathbf{W}_{1, p}^{R}[\mu](x)$ is the Wolff potential of $\mu$.

Given $r>0$, we consider a ball $B(x, 2 r) \subset \Omega$ and shrinking balls $B_{j}:=B_{r_{j}}\left(x_{0}\right)$, where $r_{j}=r 2^{-j}$ with $j \geq 0$ is an integer.

Proposition 8 (see Lemma 2.5 in [3]). Let $\mu$ be locally finite nonnegative measures on $\Omega$. Then, there exists a constant $C=$ $C(N, p)>0$ such that for any $s>0$ we have

$$
\phi^{s+1} \leq(s+1) \mathbf{W}_{1, p}^{R}\left(\phi^{s(p-1)} d \sigma\right)
$$

where $\phi=\sum_{j=0}^{\infty} c_{j} \chi_{B_{j}}$ with $c_{j}=C\left[\sigma\left(B_{j}\right) /\left(r 2^{-j}\right)^{N-p}\right]^{1 /(p-1)}$.

The following theorem is an analogue of the above theorems for $k$-Hessian equations. For more details, see [14].

Proposition 9. If $u \geq 0$ is such that $-u$ is $k$-convex in $\Omega$, then

$$
\left[\frac{\mu\left(B_{R / 8}\right)}{R^{N-2 k}}\right]^{1 / k} \leq C(n, k) \inf _{B_{R / 8}} u,
$$

for all balls $B_{R}$, provided that $B_{3 R} \subset \Omega$; here, $\mu=F_{k}[-u]$ is the corresponding $k$-Hessian measure associated with the $k$-convex function $-u$.

Proposition 10. Let $u \geq 0$ be such that $-u$ is $k$-convex in $\Omega$. Then, there is a constant $C=C(n, k)>0$ such that if $\mu=$ $F_{k}[-u]$ then

$$
C \mathbf{W}_{2 k /(k+1), k+1}^{R / 8} \mu(x) \leq u(x),
$$

whenever the ball $B_{3 R} \subset \Omega$.

\section{Proof of Theorems 1 and 2}

In this section, we will give the proof of our main theorem. Firstly, we prove the following integral estimate for solutions of quasilinear equations (1), which shows that if (1) has a nontrivial $p$-superharmonic supersolution, then $\omega$ is absolutely continuous with respect to $\sigma$. The fact can be used to obtain a characterization of removable singularities for the homogeneous quasilinear equation:

$$
-\Delta_{p} u=\sigma P(u)
$$

$$
u \geq 0, u \text { is } p \text {-superharmonic in } \Omega,
$$

in terms of Hausdorff measures. For more details, see Theorem 2.18 in [4] and Theorem 3.1 in [3].

Lemma 11. Let $\sigma$ and $\omega$ be locally finite nonnegative measures on $\Omega$ and $p>1$. There exists a constant $C=C(N, p, \alpha, \beta, \ell)>$ 0 such that if $u(x)$ is a solution to (1), then

$$
\begin{aligned}
& \int_{B_{R}} P_{\ell, \alpha, \beta}(u) d \sigma+\omega\left(B_{R}\right) \\
& \quad \leq C \sigma\left(B_{R}\right)^{(p-1) /(\ell \beta-p+1)} R^{\ell \beta(N-p) /(\ell \beta-p+1)},
\end{aligned}
$$

for all balls $B_{R}$ such that $B_{2 R} \subset \Omega$.

Proof. Define

$$
d \mu=\sigma P_{\ell, \alpha, \beta}(u)+\omega .
$$

According to Proposition 6 and the definitions of $P_{\ell, \alpha, \beta}$, we know that, for all $x \in B_{R}$,

$$
\begin{aligned}
\frac{\alpha^{\ell}}{\ell !}\left[\frac{\mu\left(B_{R}\right)}{R^{N-p}}\right]^{\ell \beta /(p-1)} & \leq P_{\ell, \alpha, \beta}\left(\left[\frac{\mu\left(B_{R}(x)\right)}{R^{N-p}}\right]^{1 /(p-1)}\right) \\
& \leq C P_{\ell, \alpha, \beta}(u)(x) .
\end{aligned}
$$

Integrating both sides of (21) against $d \sigma$ over $B_{R}$, we find

$$
\frac{\alpha^{\ell}}{\ell !} \sigma\left(B_{R}\right)\left[\frac{\mu\left(B_{R}\right)}{R^{N-p}}\right]^{\ell \beta /(p-1)} \leq C \int_{B_{R}} P_{\ell, \alpha, \beta}(u)(x) d \sigma,
$$

which combined with (20) implies that

$$
\frac{\alpha^{\ell}}{\ell !} \sigma\left(B_{R}\right)\left[\frac{\mu\left(B_{R}\right)}{R^{N-p}}\right]^{\ell \beta /(p-1)} \leq C \mu\left(B_{R}\right)
$$

This inequality is equivalent to

$$
\mu\left(B_{R}\right) \leq C \sigma\left(B_{R}\right)^{(p-1) /(\ell \beta-p+1)} R^{\ell \beta(N-p) /(\ell \beta-p+1)},
$$

which, together with (20), leads to (19).

Proof of Theorem 1. For fixed $x_{0} \in \Omega$, let $R>0$ be such that $B_{4 R}\left(x_{0}\right) \subset \Omega$. Suppose that $u$ is a positive solution of (1). In 
view of the lower pointwise potential estimate (14), we find that, for all $x \in B_{R}\left(x_{0}\right)$,

$$
\begin{aligned}
u(x) & \geq C \mathbf{W}_{1, p}^{R}[\omega](x), \\
u(x) & \geq C \mathbf{W}_{1, p}^{R}\left(P_{\ell, \alpha, \beta}(u) d \sigma\right)(x) \\
& \geq C \mathbf{W}_{1, p}^{R}\left(\frac{\alpha^{\ell}}{\ell !} u^{\ell \beta} d \sigma\right)(x) \\
& =C\left(\frac{\alpha^{\ell}}{\ell !}\right)^{1 /(p-1)} \mathbf{W}_{1, p}^{R}\left(u^{\ell \beta} d \sigma\right)(x),
\end{aligned}
$$

where $C$ depends on $N, p$.

Restrict the integration $\sigma$ on $B_{R}\left(x_{0}\right)$ and let $d \sigma^{\prime}=$ $\chi_{B_{R}\left(x_{0}\right)} d \sigma$; thus, taking into account (25), we obtain

$$
\begin{gathered}
u(x) \geq C\left(\frac{\alpha^{\ell}}{\ell !}\right)^{1 /(p-1)} \mathbf{W}_{1, p}^{R}\left(\left(C \mathbf{W}_{1, p}^{R}[\omega]\right)^{\ell \beta} d \sigma\right)(x) \\
=C^{1+\ell \beta /(p-1)}\left(\frac{\alpha^{\ell}}{\ell !}\right)^{1 /(p-1)} \int_{0}^{R}\left(\frac{1}{t^{N-p}}\right. \\
\left.\cdot \int_{B_{t}(x) \cap B_{r}\left(x_{0}\right)}\left(\mathbf{W}_{1, p}^{R}[\omega](y)\right)^{\ell \beta} d \sigma(y)\right)^{1 /(p-1)} \frac{d t}{t}
\end{gathered}
$$

in view of

$$
\begin{aligned}
\mathbf{W}_{1, p}^{R}[\omega](y) \geq & C_{0}(N, p) \chi_{B_{r}\left(x_{0}\right)}(y) \\
& \cdot \int_{r}^{R / 2}\left(\frac{\omega\left(B_{s}\left(x_{0}\right)\right)}{s^{N-p}}\right)^{1 /(p-1)} \frac{d s}{s},
\end{aligned}
$$

which combined with (26) leads to the fact that, for all $x \in$ $B_{R}\left(x_{0}\right)$,

$$
\begin{aligned}
u(x) \geq & C^{1+\ell \beta /(p-1)}\left(\frac{\alpha^{\ell}}{\ell !}\right)^{1 /(p-1)}\left[C_{0} M\left(x_{0}, \omega\right)\right]^{\ell \beta /(p-1)} \\
& \cdot \mathbf{W}_{1, p}^{R}\left(\chi_{B_{R}\left(x_{0}\right)} d \sigma\right)(x)
\end{aligned}
$$

where $M\left(x_{0}, \omega\right)$ is defined as

$$
M\left(x_{0}, \omega\right)=\int_{r}^{R / 2}\left(\frac{\omega\left(B_{s}\left(x_{0}\right)\right)}{s^{N-p}}\right)^{1 /(p-1)} \frac{d s}{s} .
$$

Thus, taking into account (26) and (28) and arguing by induction, we find

$$
\begin{aligned}
& u\left(x_{0}\right) \\
& \quad \geq C(N, p, \alpha, \beta, \ell)\left[M\left(x_{0}, \omega\right)\right]^{[\ell \beta /(p-1)]^{n}} \mathfrak{M}^{n} \mathbf{1}\left(x_{0}\right),
\end{aligned}
$$

where $\mathfrak{M}$ is a nonlinear integral operator defined by $\mathfrak{M} f=$ $\mathbf{W}_{1, p}^{R}\left(f^{\ell \beta} d \sigma^{\prime}\right)$. The iterates of $\mathfrak{M}$ are denoted by $\mathfrak{M}^{i} f=$ $\mathfrak{M}\left(\mathfrak{M}^{i-1} f\right)$. It is then easy to see from Proposition 8 that, for all $s>0$,

$$
\begin{aligned}
& \mathfrak{M} \mathbf{1}\left(x_{0}\right)=\mathbf{W}_{1, p}^{R}\left(d \sigma^{\prime}\right) \geq \phi(y), \\
& \mathfrak{M}\left(\phi^{s(p-1)}\right)(y) \geq \frac{\phi^{s \ell \beta+1}(y)}{s \ell \beta+1},
\end{aligned}
$$

where $\phi(y)$ appears in Proposition 8. Consequently,

$$
\begin{gathered}
\mathfrak{M}^{n} \mathbf{1}\left(x_{0}\right) \geq \prod_{j=1}^{n-1}\left\{\sum_{i=1}^{j}\left[\frac{\ell \beta}{p-1}\right]^{i}\right\}^{-[\ell \beta /(p-1)]^{n-j-1}} \\
\cdot\left(\phi\left(x_{0}\right)\right)^{\sum_{i=0}^{n-1}[\ell \beta /(p-1)]^{i}},
\end{gathered}
$$

and this yields

$$
\begin{aligned}
& \underset{n \rightarrow \infty}{\limsup }\left[\mathfrak{M}^{n} \mathbf{1}\left(x_{0}\right)\right]^{[(p-1) / \ell \beta]^{n}} \\
& \quad \geq C(N, p, \ell, \alpha, \beta)\left(\phi\left(x_{0}\right)\right)^{(p-1) /(\ell \beta-p+1)} .
\end{aligned}
$$

Here, we use the fact that

$$
\sum_{i=1}^{\infty}\left[\frac{p-1}{\ell \beta}\right]^{i}=\frac{p-1}{\ell \beta-p+1}, \quad \text { if } \ell \beta>p-1 .
$$

In the following, we will divide the proof into two cases.

Case $1\left(u\left(x_{0}\right)<\infty\right)$. In this case, (33), combined with (30), implies that

$$
1 \geq C(N, p, \ell, \alpha, \beta) M\left(x_{0}, \omega\right)\left(\phi\left(x_{0}\right)\right)^{(p-1) /(\ell \beta-p+1)} .
$$

Recalling that,

$$
\begin{aligned}
\phi\left(x_{0}\right) & =\sum_{j=0}^{\infty} c_{j}=\sum_{j=0}^{\infty} C\left[\frac{\sigma\left(B_{r 2^{-j}}\left(x_{0}\right)\right)}{\left(r 2^{-j}\right)^{N-p}}\right]^{1 /(p-1)} \\
& \geq C_{2}(N, p) \int_{0}^{r}\left[\frac{\sigma\left(B_{t}\left(x_{0}\right)\right)}{t^{N-p}}\right]^{1 /(p-1)} \frac{d t}{t},
\end{aligned}
$$

which leads to (7).

Case $2\left(u\left(x_{0}\right)=\infty\right)$. According to [12], we know that $u<\infty$ a.e. in $\Omega$. Therefore, choose a sequence $\left\{x_{n}\right\}_{n \geq 1} \subset B_{R / 10}\left(x_{0}\right)$, such that $\lim _{n \rightarrow \infty} x_{n}=x_{0}$ and $u\left(x_{n}\right)<\infty$. Then, (7) holds with $x_{n}$ in place of $x_{0}$, for all $n \geq 1$. Then, (7) holds by the lower semicontinuity of Wolff potentials inequality.

The proof of inequality (8) is completely similarly and more details are omitted.

Proof of Theorem 2. Let $u$ be a nonnegative $p$-superharmonic of (1). Then, $u$ satisfies (7), while it is well known that

$$
\int_{0}^{r}\left[\frac{\sigma\left(B_{t}\right)}{t^{N-p}}\right]^{1 /(p-1)} \frac{d t}{t}=\infty
$$

Provided that $d \sigma=|x|^{-r} d x$ with $r>p$, which contradicts (7).

\section{Proof of Theorems 3 and 4}

In this section, we will prove Theorem 3. It is interesting to note that, in order to prove this theorem, we should give a new iterative process. 
Proof of Theorem 3. This proof will be divided into two parts according to the value of $p$.

Case $1(1<p \leq 2)$. For nonnegative measurable functions $f$, define

$$
\mathcal{N}(f)(x)=\mathbf{W}_{1, p}^{R}\left(f^{p-1} d \sigma^{\prime}\right)(x) .
$$

Obviously, $\mathcal{N}$ is a homogeneous superlinear operator acting on nonnegative functions. Assume that $u$ is a solution of (1); then, for all $x \in B_{R}\left(x_{0}\right)$,

$$
\begin{aligned}
u(x) & \geq C \mathbf{W}_{1, p}^{R}\left(u^{p-1} d \sigma^{\prime}\right)(x)+C \mathbf{W}_{1, p}^{R}[\omega](x) \\
& =C \mathscr{N}(u)(x)+C \mathbf{W}_{1, p}^{R}[\omega](x) \\
& \geq C^{2} \mathcal{N}\left(\mathbf{W}_{1, p}^{R}[\omega]\right)(x)+C \mathbf{W}_{1, p}^{R}[\omega](x),
\end{aligned}
$$

where $C$ depends on $N, p$.

Iterating (39) $n$ times yields

$$
u(x) \geq \sum_{i=1}^{i=n} C^{i+1} \mathcal{N}^{i}\left(\mathbf{W}_{1, p}^{R}[\omega]\right)(x)+C \mathbf{W}_{1, p}^{R}[\omega](x) .
$$

Here, we use the fact that $\mathcal{N}$ is a homogeneous superlinear operator and $i$ th iterate of $\mathcal{N}$ is defined by $\mathcal{N}^{i}(u)=$ $\mathcal{N}\left(\mathcal{N}^{i-1}(u)\right)$ for $i>1$.

In the following, we will estimate the iterates of $\mathcal{N}$. Recall $d \sigma^{\prime}=\chi_{B_{R}\left(x_{0}\right)} d \sigma ;$ thus,

$$
\begin{aligned}
& \mathcal{N}\left(\mathbf{W}_{1, p}^{R}[\omega]\right)(x)=\int_{0}^{R}\left(\frac{1}{t^{N-p}}\right. \\
& \left.\cdot \int_{B_{t}(x) \cap B_{r}\left(x_{0}\right)}\left(\mathbf{W}_{1, p}^{R}[\omega](y)\right)^{p-1} d \sigma(y)\right)^{1 /(p-1)} \frac{d t}{t},
\end{aligned}
$$

in view of

$$
\mathbf{W}_{1, p}^{R}[\omega](y) \geq C_{0}(N, p) M\left(x_{0}, \omega\right),
$$

where $M\left(x_{0}, \omega\right)$ is defined in (29). Consequently, for all $x \in$ $B_{R}\left(x_{0}\right)$,

$$
\begin{aligned}
\mathscr{N} & \left(\mathbf{W}_{1, p}^{R}[\omega]\right)(x) \\
& \geq C_{0} M\left(x_{0}, \omega\right) \mathbf{W}_{1, p}^{R}\left(\chi_{B_{R}\left(x_{0}\right)} d \sigma\right)(x) \\
& \geq C_{0} M\left(x_{0}, \omega\right) \phi(y),
\end{aligned}
$$

where $\phi(y)$ appears in Proposition 8. Obviously,

$$
\mathscr{N}^{i}\left(\mathbf{W}_{1, p}^{R}[\omega]\right)(x) \geq\left[C_{0} M\left(x_{0}, \omega\right)\right]^{i} \frac{\phi^{i}(y)}{i !} .
$$

Here, the following fact has been used in this inequality:

$$
\mathcal{N}\left(\phi^{s(p-1)}\right)(y) \geq \frac{\phi^{s(p-1)+1}(y)}{s(p-1)+1} .
$$

Note that $i$ here is arbitrary; this fact, together with (40) and (44), leads to

$$
\begin{aligned}
u\left(x_{0}\right) & \geq \sum_{i=0}^{i=\infty} \frac{\left[C C_{0} M\left(x_{0}, \omega\right) \phi(y)\right]^{i}}{i !} \\
& =\exp \left\{C C_{0} M\left(x_{0}, \omega\right) \phi(y)\right\},
\end{aligned}
$$

which, combined with (36), leads to (9) provided that $u\left(x_{0}\right)<$ $\infty$. In a similar way, we can prove (9) if $u\left(x_{0}\right)=\infty$; more details are omitted.

Case $2(p>2)$. A point worth emphasizing is that the operator $\mathcal{N}$ defined by (38) does not fall within this framework since it is not a superlinear operator. Therefore, define

$$
\mathcal{N}(f)(x)=\left(\mathbf{W}_{1, p}^{R}\left(f d \sigma^{\prime}\right)\right)^{p-1}(x) .
$$

In this case, we have

$$
\begin{aligned}
u(x)^{p-1} & \geq C^{p-1}\left[\mathbf{W}_{1, p}^{R}[\omega]\right]^{p-1}(x) \\
& \geq\left(C C_{0} M\left(x_{0}, \omega\right)\right)^{p-1}, \\
u(x) & \geq C \mathbf{W}_{1, p}^{R}\left(u^{p-1} d \sigma\right)(x)+C \mathbf{W}_{1, p}^{R}[\omega](x) .
\end{aligned}
$$

Thus, by Minkowski's inequality,

$$
\begin{aligned}
u(x)^{p-1} \geq & C^{p-1}\left[\mathbf{W}_{1, p}^{R}\left(u^{p-1} d \sigma\right)\right]^{p-1}(x) \\
& +\left[C \mathbf{W}_{1, p}^{R}[\omega]\right]^{p-1} \\
= & C^{p-1} \mathcal{N}\left(u^{p-1}\right)(x)+\left[C \mathbf{W}_{1, p}^{R}[\omega]\right]^{p-1}(x) \\
\geq & C^{2(p-1)} \mathcal{N}\left\{\left(\mathbf{W}_{1, p}^{R}[\omega]\right)^{p-1}\right\}(x) \\
& +\left[C \mathbf{W}_{1, p}^{R}[\omega]\right]^{p-1},
\end{aligned}
$$

where $C$ depends on $N, p$. It is clear that

$$
\begin{aligned}
\mathcal{N} & \left\{\left(\mathbf{W}_{1, p}^{R}[\omega]\right)^{p-1}\right\}(x)=\left[\int _ { 0 } ^ { R } \left(\frac{1}{t^{N-p}}\right.\right. \\
& \left.\left.\cdot \int_{B_{t}(x) \cap B_{r}\left(x_{0}\right)}\left(\mathbf{W}_{1, p}^{R}[\omega]\right)^{p-1} d \sigma(y)\right)^{1 /(p-1)} \frac{d t}{t}\right]^{p-1} \\
& \geq\left[C_{0} M\left(x_{0}, \omega\right) \mathbf{W}_{1, p}^{R}\left(\chi_{B_{R}\left(x_{0}\right)} d \sigma\right)\right]^{p-1}(x) \\
& \geq\left[C_{0} M\left(x_{0}, \omega\right) \phi(y)\right]^{p-1},
\end{aligned}
$$

where $C_{0}, M\left(x_{0}, \omega\right)$ appears in (29). Using (49) and (50), we find

$$
\begin{aligned}
u(x)^{p-1} \geq & {\left[C^{2} C_{0} M\left(x_{0}, \omega\right) \phi(y)\right]^{p-1} } \\
& +\left[C C_{0} M\left(x_{0}, \omega\right) \phi(y)\right]^{p-1} .
\end{aligned}
$$


Therefore,

$$
u(x)^{p-1} \geq \sum_{i=0}^{i=\infty}\left[\frac{\left[C^{2} C_{0} M\left(x_{0}, \omega\right) \phi(y)\right]^{i}}{i !}\right]^{p-1} .
$$

By reverse Hölder inequality, we get

$$
\begin{aligned}
u(x) & \geq \sum_{i=0}^{i=\infty} \frac{\left[C^{2} C_{0} M\left(x_{0}, \omega\right) \phi(y)\right]^{i}}{i !} \\
& =\exp \left\{C^{2} C_{0} M\left(x_{0}, \omega\right) \phi(y)\right\} .
\end{aligned}
$$

The following proof is similar to that of (46), so it is clear.

This finishes the proof of Theorem 3.

The proof of Theorem 4 is standard and will be omitted.

\section{A Fully Nonlinear Analogue: The $k$-Hessian}

We now move to $k$-Hessian operator and present fully nonlinear counterparts of the results obtained in the previous theorems. More precisely, consider fully nonlinear $k$-Hessian operator $F_{k}$, introduced by Trudinger and Wang [15-17]:

$$
F_{k}[-u]=\sigma P(u)+\omega, \quad u \geq 0,-u \text { is } k \text {-convex in } \Omega,
$$

where $F_{k}[u]$ denotes the $k$-Hessian $(k=1,2, \ldots, n)$,

$$
F_{k}[u]=\sum_{1 \leq i_{1}<i_{2}<\cdots<i_{k} \leq n} \lambda_{i_{1}} \cdots \lambda_{i_{k}}
$$

where $\lambda_{i_{1}} \cdots \lambda_{i_{k}}$ are the eigenvalues of the Hessian matrix $D^{2} u$; that is, $F_{k}[u]$ is the sum of the $k \times k$ principal minors of $D^{2} u$, which coincides with the Laplacian $F_{1}[u]=\Delta_{p} u$ if $k=1$, and the Monge-Ampère operator $F_{n}[u]=\operatorname{det}\left(D^{2} u\right)$ if $k=n$.

The proof of the following theorems is completely analogous to that of (1). One only needs to use Propositions 9 and 10 in place of Propositions 6 and 7, respectively, and argue as in Sections 3 and 4 with $\mathbf{W}_{2 k /(k+1), k+1}^{R}$ in place of $\mathbf{W}_{1, p}^{R}$. Therefore, the proof is omitted.

Theorem 12. Let $u(x)$ be a solution of (54) in $\Omega$ with $p>1$ and $\ell \beta>k$. Suppose that $B_{4 R}\left(x_{0}\right) \subset \Omega$. Then, there exists a constant $M=M(N, p, \alpha, \beta, \ell)$ such that

$$
\begin{gathered}
\left\{\int_{0}^{r}\left[\frac{\sigma\left(B_{t}\right)}{t^{N-p}}\right]^{1 / k} \frac{d t}{t}\right\}^{k /(\ell \beta-k)} \\
\cdot \int_{r}^{R / 16}\left[\frac{\omega\left(B_{t}\left(x_{0}\right)\right)}{t^{N-p}}\right]^{1 / k} \frac{d t}{t} \leq M, \\
\left\{\int_{0}^{r}\left[\frac{\sigma\left(B_{t}\right)}{t^{N-p}}\right]^{1 / k} \frac{d t}{t}\right\}^{k /(\ell \beta-k)} \\
\cdot \int_{r}^{R / 16}\left[\frac{\int_{B_{t}} P_{\ell, \alpha, \beta}(u) d \sigma}{t^{N-p}}\right]^{1 / k} \frac{d t}{t} \leq M,
\end{gathered}
$$

for all $0<r \leq R / 32$.
Theorem 13. Let $u(x)$ be a solution of (54) in $\Omega$ with $p>1$ and $P(u)=\sigma u^{p-1}$. Suppose that $B_{4 R}\left(x_{0}\right) \subset \Omega$. Then, there exists a constant $M=M(N, p, \alpha, \beta, \ell)$ such that

$$
\begin{aligned}
& \int_{0}^{r}\left[\frac{\sigma\left(B_{t}\right)}{t^{N-p}}\right]^{1 / k} \frac{d t}{t} \int_{r}^{R / 16}\left[\frac{\omega\left(B_{t}\left(x_{0}\right)\right)}{t^{N-p}}\right]^{1 / k} \frac{d t}{t} \leq M, \\
& \int_{0}^{r}\left[\frac{\sigma\left(B_{t}\right)}{t^{N-p}}\right]^{1 / k} \frac{d t}{t} \int_{r}^{R / 16}\left[\frac{\int_{B_{t}} u^{p-1} d \sigma}{t^{N-p}}\right]^{1 / k} \frac{d t}{t} \leq M,
\end{aligned}
$$

for all $0<r \leq R / 32$.

\section{Competing Interests}

The authors declare that they have no competing interests.

\section{Acknowledgments}

This work was partially supported by the National Natural Science Foundation of China (no. 11401473 and no. 11326100), Natural Science Foundation of Gansu Province (no. 145RJZA214 and no. 1506RJYA272), Fundamental Research Funds for the Central Universities (no. 31920160059), Science and Humanity Foundation of the Ministry of Education (no. 15YJA880085), Foundation of State Nationalities Affairs Commission (no. 14XBZ016), and Innovative Team Subsidize of Northwest University for Nationalities.

\section{References}

[1] M.-F. Bidaut-Véron, N. Quoc-Hung, and L. Véron, "Quasilinear Lane-Emden equations with absorption and measure data," Journal de Mathématiques Pures et Appliquées, vol. 102, no. 2, pp. 315-337, 2014.

[2] N. Quoc-Hung and L. Véron, "Quasilinear and Hessian type equations with exponential reaction and measure data," Archive for Rational Mechanics and Analysis, vol. 214, pp. 235-267, 2014.

[3] N. C. Phuc and I. E. Verbitsky, "Local integral estimates and removable singularities for quasilinear and Hessian equations with nonlinear source terms," Communications in Partial Differential Equations, vol. 31, no. 10-12, pp. 1779-1791, 2006.

[4] N. C. Phuc and I. E. Verbitsky, "Quasilinear and Hessian equations of Lane-Emden type," Annals of Mathematics, vol. 168, pp. 859-914, 2008.

[5] N. C. Phuc and I. E. Verbitsky, "Singular quasilinear and Hessian equations and inequalities," Journal of Functional Analysis, vol. 256, no. 6, pp. 1875-1906, 2009.

[6] B. J. Jaye and I. E. Verbitsky, "Local and global behaviour of solutions to nonlinear equations with natural growth terms," Archive for Rational Mechanics and Analysis, vol. 204, no. 2, pp. 627-681, 2012.

[7] B. J. Jaye and I. E. Verbitsky, "The fundamental solution of nonlinear operators with natural growth terms," Annali della Scuola Normale Superiore di Pisa, vol. 12, pp. 93-139, 2013.

[8] C. T. Dat and I. E. Verbitsky, "Finite energy solutions of quasilinear elliptic equations with sub-natural growth terms," Calculus of Variations and Partial Differential Equations, vol. 52, no. 3, pp. 529-546, 2015. 
[9] D. T. Cao and I. E. Verbitsky, "Nonlinear elliptic equations and intrinsic potentials of Wolff type," http://arxiv.org/abs/1409 .4076 .

[10] E. Mitidieri and S. I. Pohozaev, "Nonexistence of positive solutions for quasilinear elliptic problems on $\mathbb{R}^{n}$," Proceedings of the Steklov Institute of Mathematics, vol. 277, pp. 1-32, 1999.

[11] J. Serrin and H. Zou, "Cauchy-Liouville and universal boundedness theorems for quasilinear elliptic equations and inequalities," Acta Mathematica, vol. 189, no. 1, pp. 79-142, 2002.

[12] J. Heinonen, T. Kilpeläinen, and O. Martio, Nonlinear Potential Theory of Degenerate Elliptic Equations, Oxford University Press, Oxford, UK, 1993.

[13] T. Kilpeläinen and J. Malý, "Degenerate elliptic equations with measure data and nonlinear potentials," Annali della Scuola Normale Superiore di Pisa-Classe di Scienze, vol. 19, no. 4, pp. 591-613, 1992.

[14] D. A. Labutin, "Potential estimates for a class of fully nonlinear elliptic equations," Duke Mathematical Journal, vol. 111, no. 1, pp. $1-49,2002$.

[15] N. S. Trudinger and X.-J. Wang, "Hessian measures I," Topological Methods in Nonlinear Analysis, vol. 10, no. 2, pp. 225-239, 1997.

[16] N. S. Trudinger and X. J. Wang, "Hessian measures II," Annals of Mathematics, vol. 150, pp. 579-604, 1999.

[17] N. S. Trudinger and X.-J. Wang, "Hessian measures III," Journal of Functional Analysis, vol. 193, no. 1, pp. 1-23, 2002. 


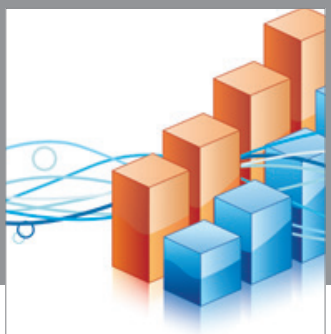

Advances in

Operations Research

vatem alat4

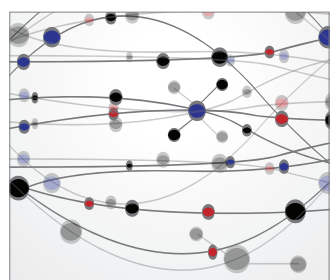

\section{The Scientific} World Journal
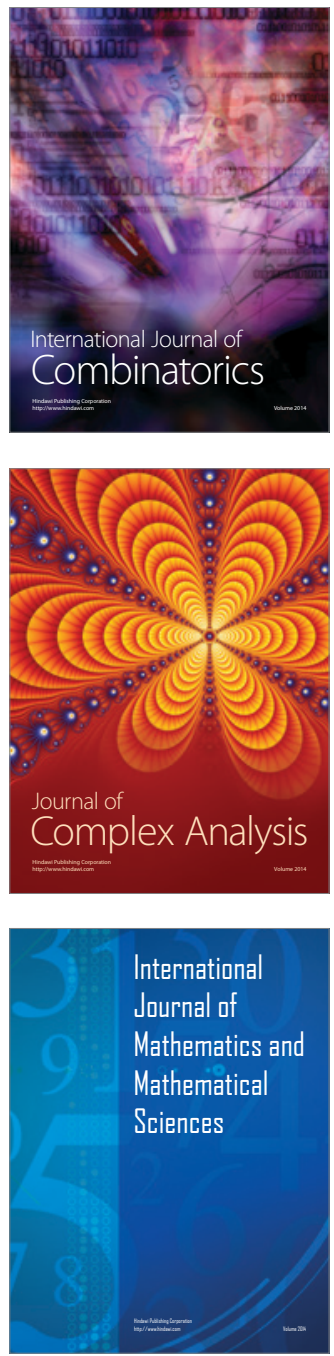
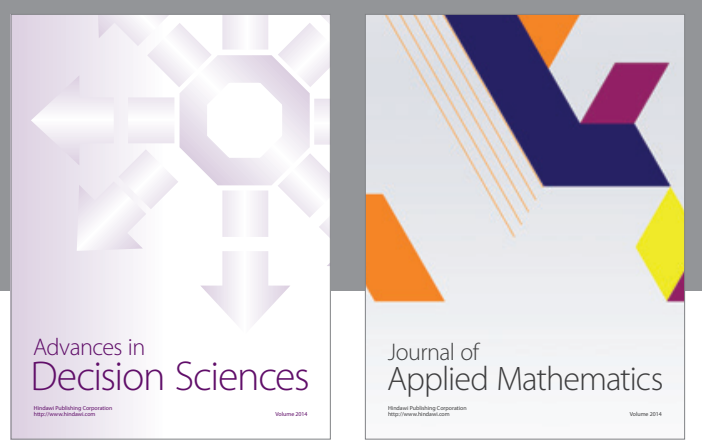

Algebra

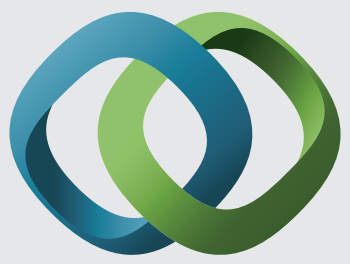

\section{Hindawi}

Submit your manuscripts at

http://www.hindawi.com
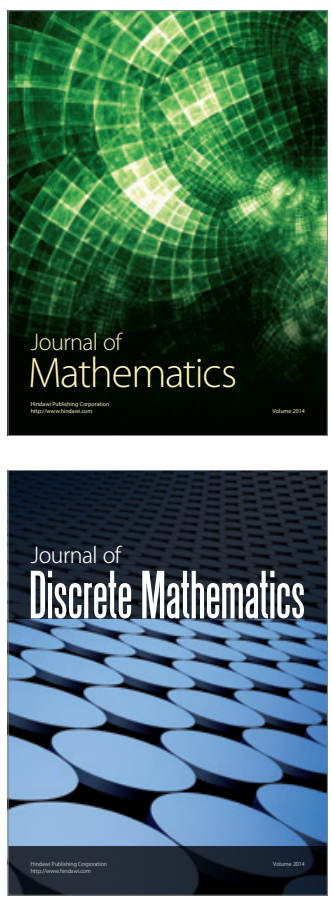

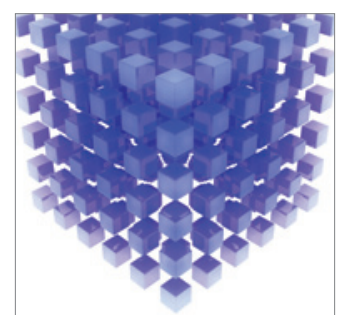

Mathematical Problems in Engineering
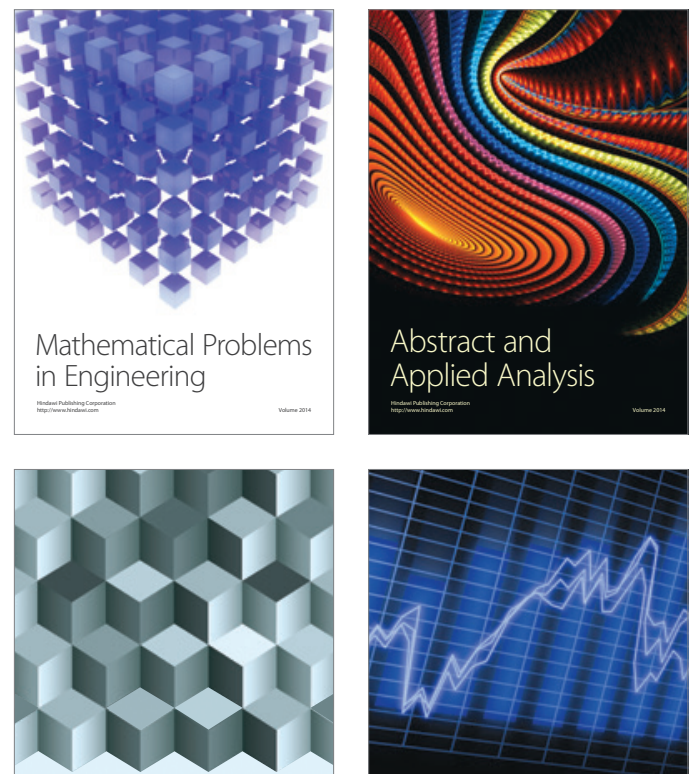

Journal of

Function Spaces

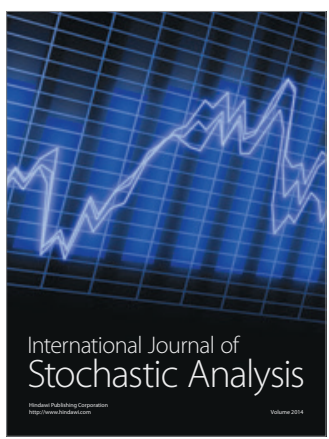

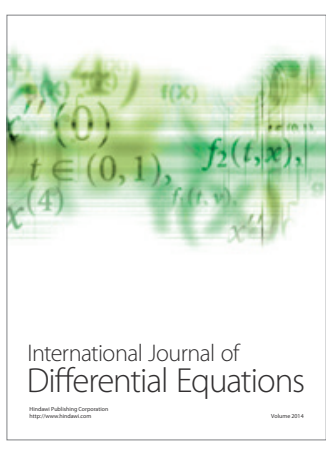
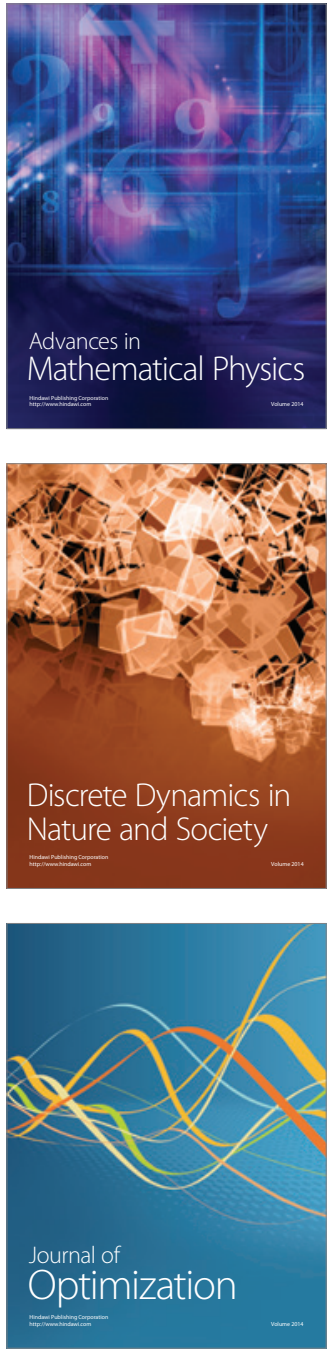